\title{
Study of Atrial Fibrillation and Venous Thromboembolism in Critically Ill Patients in Intensive Care Unit
}

Yasser Elsayed Mohammed

Cardiology Department, Faculty of Medicine, Al-Azhar University

\begin{abstract}
:
Bachground: atrial fibrillation (AF) is the most common arrhythmia in patient hospitalized in ICU and is associated with increased morbidity and mortality. Venous Thromboembolism (VTE), including deep venous thrombosis (DVT) and pulmonary Embolism (PE), is recognized as a common complication in critically ill patients. Risk factors including critical illness, mechanical ventilation, sedative medications and central venous catheter insertion are major contributing factors to the high risk of VTE. Objective: it was to evaluate the causes and outcomes of AF and VTE in patients admitted to intensive care unit due to non-cardiac causes. We studied 143 consecutive patients admitted to ICU for non-cardiac emergencies. Results: only $48(33.5 \%)$ patients were complicated. AF is the most common complication happened in 27 patients $(56 \%)$ of complicated cases; pulmonary embolism comes next with 13 patients $(27.1 \%)$ of complicated cases, DVT in the third place with 8 patients (16.6 $\%)$ of complicated cases. The age of patients admitted to ICU was directly related to complication happened in ICU, the older the patient is the more he is vulnerable to complications, the increased body mass index and increased weight was directly related to complications, the hemoglobin level is strongly related to cardiac complications. There was increased risk of DVT and pulmonary embolism due to prolonged bed ridden state.

Conclusion: patients of ICU who have a higher risk than other for adverse cardiac complications, the most common cardiac complications are AF, DVT and pulmonary embolism and finally cardiac complications leads to increased rate of mortality and morbidity.
\end{abstract}

Keywords: Atrial Fibrillation, Venous Thromboembolism, Intensive Care Unit.

\section{Introduction:}

Atrial fibrillation (AF) is the most common arrhythmia in patients hospitalized in intensive care units (ICUs) and is associated with increased morbidity and mortality. Most frequently, the diagnosis of the underlying arrhythmia is possible from the physical examination, the response to maneuvers or drugs, and the 12-lead surface electrocardiogram ${ }^{(1)}$.

Twelve leads electrocardiogram is the corner stone to identify the rhythm of tachyarrhythmia, presence of pre-excitation, Brady arrhythmias and heart blocks. ${ }^{(2)}$.

Cardiac arrhythmias are a common problem encountered in the intensive care unit (ICU) and represent a major source of morbidity. The inciting factor for an arrhythmia in a given patient may be an insult such as hypoxia, infection, cardiac ischemia, catecholamine excess (endogenous or exogenous), or an electrolyte abnormality ${ }^{(3)}$.

Venous thromboembolism (VTE), including deep venous thrombosis (DVT) and pulmonary embolism (PE), is recognized as a common complication in critically ill patients. Risk factors including critical illness, mechanical ventilation, sedative medications, and central venous catheter insertion are major contributing factors to the high risk of VTE ${ }^{(1)}$.

VTE is a common lethal disease that affects hospitalized patients, recurs frequently, is often overlooked, and can result in long term complications, including chronic thromboembolic pulmonary hypertension and post thrombotic syndrome. Although it is the 3rd most common cause of hospital-related deaths, less than half of all hospitalized patients at risk for VTE, received adequate prophylactic treatment. Most of hospitalized patients have at least one or more risk factors for VTE, and without prophylaxis, the incidence of hospital acquired DVT is $10 \%$ to $20 \%$ among medical patients and even higher among surgical patients ( $15 \%$ to $40 \%)^{(4)}$

\section{Patients and Methods:}

The study included 143 consecutive patients who admitted to ICU for non cardiac emergencies. The study was approved by the Ethics Board of Al-Azhar University.

All patients admitted to ICU, from November 2015 to november 2016 were included in the study, 319 patients were admitted to ICU during this time with medical emergency, surgical emergency or for postoperative observation, 167 patients were 
excluded from the study. The remaining 143 patients were enrolled in the study as they were admitted due to non-cardiac etiology.

\section{Exclusion criteria:}

All patients admitted due to cardiac emergencies such as cardiogenic shock ,acute coronary syndrome, symptomatic bradycardia or tachycardia, Heart failure, acute pulmonary edema , Cardiac temponade, acute pulmonary embolism, duration of stay in ICU less than 3 days and Patient with chronic AF.

The remaining 143 patients fulfilled the following inclusion criteria were included with emphasis on duration of stay in ICU to be at least 3 days or more.

\section{Inclusion criteria:}

The study included all 143 patients admitted to ICU due tomedical causes as cerebro-vascular accidents, chest problems and respiratory failure,endocrinal or metabolic abnormalities, acute renal failure or chronic renal failure, liver cell failure, or surgical causes as road traffic accidents, multiple traumas, acute pancreatitis, acute cholecystitis and other acute abdomen emergencies and Postoperative causes as after neurosurgical operations e.g. excision of brain tumor and after orthopedic operations e.g. total hip replacement surgery.

Clinical assessment of all patients, age, gender, body weight, height $\mathrm{m}$ body mass index, heart rate ,temperature, respiratory rate , duration of stay. Risk factors as Diabetes mellitus which was defined as fasting plasma glucose $\geq 126 \mathrm{mg} / \mathrm{dl}$ and/or random plasma glucose $\geq 200 \mathrm{mg} / \mathrm{dl}$ and/or using of glucose lowering medication. Duration and type of medication (oral hypoglycemic drugs, Insulin or combination of both) were also noted. Hypertension defined as blood pressure BP $>140 / 90$, and/or use of BP lowering medication. Dyslipidemia defined as total cholesterol > $200 \mathrm{mg} / \mathrm{dL}$, LDL cholesterol > 100 $\mathrm{mg} / \mathrm{dL}$, use of lipid lowering medication or hypertiglyceremia defined as TAGs $>150 \mathrm{mg}$ /dL. Smokers were defined as those who currently smoking or had quit within the last 5 years.

Laboratory work up to all patients, including complete blood count, fasting lipid profile (total cholesterol, LDL, and TGs), sodium, Potassium, Magnesium and Calcium levels in blood International normalization ratio, arterial blood gases, liver function tests (AST - ALT) and Kidney function tests (blood urea \& Creatinine).

Echocardiography was done to all patients ,two-dimensional, M-mode and Doppler echocardiographic studies using a 2.5 $\mathrm{MHz}$ transducer of the commercially available machine within 2-3 days after ICU admission.

Statistical Analysis:

Data were collected, revised, coded and entered to the Statistical Package for Social Science (IBM SPSS) version 20. The qualitative data were presented as number and percentages while quantitative data were presented as mean, standard deviations and ranges when their distribution found parametric and were presented as median with interquartile ranges (IQR) when their distribution found nonparametric.

\section{Result:}

Table (1): Demographic data and risk factors of the whole studied population.

\begin{tabular}{|c|c|c|}
\hline \multicolumn{2}{|c|}{} & No.=143 \\
\hline Age & Mean \pm SD & $60.76 \pm 16.73$ \\
& & $77(53.8 \%)$ \\
\hline Sex & Female & $66(46.2 \%)$ \\
\hline Hypertension & Male & $79(55.2 \%)$ \\
\hline DM & Yes & $82(57.3 \%)$ \\
\hline Smoking & Yes & $58(40.6 \%)$ \\
\hline Heart failure & Yes & $42(29.4 \%)$ \\
\hline
\end{tabular}


Table (2): Shows the causes of admission to ICU among the studied population.

\begin{tabular}{|l|c|c|}
\hline \multicolumn{1}{|c|}{ Cause of admission to ICU } & No & $\%$ \\
\hline Medical emergency & 128 & 89.6 \\
\hline Chest infection & 41 & 28.7 \\
\hline Stroke & 24 & 16.8 \\
\hline Diabetic ketoacidosis & 20 & 14 \\
\hline Intra-cerebral hemorrhage & 13 & 9.1 \\
\hline Septic shock & 8 & 5.6 \\
\hline Respiratory failure type 2 & 7 & 5.4 \\
\hline Brain tumor & 4 & 2.8 \\
\hline Bronchial Asthma & 3 & 2.1 \\
\hline Meningitis & 2 & 1.4 \\
\hline Disturbed Level of Consciousness & 2 & 1.4 \\
\hline Post-arrest & 2 & 1.4 \\
\hline Status Epileptics & 1 & 0.7 \\
\hline Sancer stomach & 1 & 0.7 \\
\hline Fracture femur & 15 & 10.4 \\
\hline Facture pelvis & 5 & 3.5 \\
\hline Postoperative peritonitis & 4 & 2.8 \\
\hline Run shot & 2 & 1.4 \\
\hline Road traffic accident & 3 & 2.1 \\
\hline
\end{tabular}

Table (3): Shows different vital signs, Weight in $\mathrm{Kg}$, height in $\mathrm{cm}$, body mass index and duration of stay in ICU of the studied population.

\begin{tabular}{||l|c|c||}
\hline \multicolumn{2}{|c|}{ Vital Signs } & No.=143 \\
\hline Heart Rate & Mean \pm SD & $100.66 \pm 19.25$ \\
\hline Systolic BP & Mean \pm SD & $127.90 \pm 26.82$ \\
\hline Diastolic BP & Mean \pm SD & $77.66 \pm 14.97$ \\
\hline Temperature $\left({ }^{\circ} \mathrm{C}\right)$ & Mean \pm SD & $38.06 \pm 0.59$ \\
\hline Respiratory Rate & Mean \pm SD & $24.30 \pm 4.37$ \\
\hline Weight $(\mathrm{Kg})$ & Mean \pm SD & $90.27 \pm 18.19$ \\
\hline Height $(\mathrm{Cm})$ & Mean \pm SD & $167.71 \pm 5.91$ \\
\hline Body Mass Index & Mean \pm SD & $32.05 \pm 6.44$ \\
\hline Duration of stay in ICU (days) & Mean \pm SD & $8.21 \pm 2.91$ \\
\hline
\end{tabular}

Table (4): Shows the number of complicated patients, types of complications and fate of all studied population.

\begin{tabular}{||c|l|c|c|}
\hline \multicolumn{2}{|c|}{ No. } & $\%$ \\
\hline \multirow{2}{*}{ Complications } & Non complicated & 95 & $66.43 \%$ \\
& Complicated & 48 & $33.5 \%$ \\
\hline \multirow{2}{*}{ Types of complications } & Atrial fibrillation & 27 & $56.0 \%$ \\
& Deep venous thrombosis & 8 & $16.6 \%$ \\
& Pulmonary embolism & 13 & $27.08 \%$ \\
\hline \multirow{2}{*}{ Fate } & & 49 & $34.3 \%$ \\
\hline
\end{tabular}


Study of Atrial Fibrillation and Venous Thromboembolism....

Table (5): Shows the laboratory data collected from studied population.

\begin{tabular}{|c|c|c|}
\hline Total leucocytic count (cells/ul) & Mean \pm SD & $15292.38 \pm 514.56$ \\
\hline $\mathrm{Hb}(\mathrm{mg} / \mathrm{dl})$ & Mean \pm SD & $10.02 \pm 2.33$ \\
\hline Platelets (K/ul) & Mean \pm SD & $220.10 \pm 40.0$ \\
\hline Urea $(\mathrm{mg} / \mathrm{dl})$ & Median & $50(37-49)$ \\
\hline Creatinine (mg/dl) & Median & $1(0.9-1.1)$ \\
\hline AST (U/L) & Median & $32(25-36)$ \\
\hline ALT (U/L) & Median & $39(30-37)$ \\
\hline $\mathrm{PH}$ & Mean \pm SD & $7.32 \pm 0.13$ \\
\hline Pco2 & Mean \pm SD & $39.33 \pm 3.21$ \\
\hline $\mathrm{HCO} 3(\mathrm{mmol} / \mathrm{L})$ & Mean \pm SD & $1947 \pm 5.62$ \\
\hline $\mathrm{Na}(\mathrm{mEq} / \mathrm{L})$ & Mean \pm SD & $13335 \pm 7.53$ \\
\hline $\mathrm{K}(\mathrm{mEq} / \mathrm{L})$ & Mean \pm SD & $397 \pm 1.00$ \\
\hline $\mathrm{Mg}(\mathrm{mEq} / \mathrm{L})$ & Mean \pm SD & $2.04 \pm 0.57$ \\
\hline $\mathrm{Ca}(\mathrm{mEq} / \mathrm{L})$ & Mean \pm SD & $9.38 \pm 0.50$ \\
\hline T. cholesterol (mg/dl] & Mean \pm SD & $209.61 \pm 23.98$ \\
\hline. $\mathrm{DL}(\mathrm{mg} / \mathrm{dl})$ & Mean \pm SD & $140.31 \pm 27.12$ \\
\hline Triglyceride (mg/dl) & Median (IQR) & $180(155-211)$ \\
\hline INR & Mean \pm SD & $1.27 \pm 0.48$ \\
\hline
\end{tabular}

Table (6): Shows Electrocardiogram data.

\begin{tabular}{||c|c|c|}
\hline \multicolumn{2}{|c|}{ ECG } & No.=143 \\
\hline \multirow{2}{*}{ Rate } & Mean \pm SD & $100.91 \pm 20.66$ \\
\hline \multirow{2}{*}{ Rhythm } & Irregular & $27(18.8 \%)$ \\
& Regular & $116(81.2 \%)$ \\
\hline \multirow{2}{*}{ Ischemia } & No & $103(72.0 \%)$ \\
& Yes & $40(28.0 \%)$ \\
\hline
\end{tabular}


Table (7): Shows the Echocardiographic findings in the study population.

\begin{tabular}{|c|c|c|}
\hline \multicolumn{2}{|c|}{ ECHO findings } & \multirow{2}{*}{$\begin{array}{l}\text { No. }=143 \\
2.48 \pm 0.28\end{array}$} \\
\hline Aortic root $(\mathrm{cm})$ & Mean \pm SD & \\
\hline left atrium $(\mathrm{cm})$ & Mean \pm SD & $3.76 \pm 0.48$ \\
\hline $\mathrm{EF} \%$ & Mean \pm SD & $56.53 \pm 8.68$ \\
\hline RWMA & $\begin{array}{l}\text { No } \\
\text { Yes }\end{array}$ & $\begin{array}{l}99(69.2 \%) \\
44(30.8 \%)\end{array}$ \\
\hline \multirow[t]{2}{*}{ Diastolic dysfunction } & Grade 1 & $73(51.0 \%)$ \\
\hline & $\begin{array}{l}\text { Grade } 2 \\
\text { Normal } \\
\end{array}$ & $\begin{array}{l}36(25.1 \%) \\
34(23.7 \%)\end{array}$ \\
\hline viral valve & $\begin{array}{c}\text { Mild MR } \\
\text { Moderate MR } \\
\text { Normal } \\
\text { Trivial MR }\end{array}$ & \begin{tabular}{|l|}
$68(47.6 \%)$ \\
$13(9.1 \%)$ \\
$44(30.8 \%)$ \\
$18(12.6 \%)$ \\
\end{tabular} \\
\hline Aortic valve & $\begin{array}{c}\text { Calcific } \\
\text { Mild AR Moderate } \\
\text { AR Normal } \\
\text { Trivial AR }\end{array}$ & $\begin{array}{l}19(13.3 \%) \\
3(2.1 \%) \\
5(3.5 \%) \\
113(79.0 \%) \\
3(2.1 \%) \\
\end{array}$ \\
\hline Pulmonary valve & $\begin{array}{l}\text { Mild PR } \\
\text { Normal }\end{array}$ & $\begin{array}{l}2(1.4 \%) \\
141(98.6 \%)\end{array}$ \\
\hline Right Ventricle & $\begin{array}{l}\text { Dilated } \\
\text { Normal }\end{array}$ & \begin{tabular}{|l|}
$22(15.4 \%)$ \\
$121(84.6 \%)$
\end{tabular} \\
\hline Tricuspid valve & $\begin{array}{c}\text { Mild TR } \\
\text { Moderate TR } \\
\text { Normal } \\
\text { Severe TR } \\
\text { Trivial TR } \\
\end{array}$ & \begin{tabular}{|l|}
$24(16.8 \%)$ \\
$9(6.3 \%)$ \\
$73(51.0 \%)$ \\
$3(2.1 \%)$ \\
$34(23.8 \%)$ \\
\end{tabular} \\
\hline Pulmonary artery Pressure (mmhg) & Mean \pm SD & $31.34 \pm 11.29$ \\
\hline
\end{tabular}

Table (8): Relation between complication and demographic data and risk factors of the studied patients.

\begin{tabular}{|c|c|l|l|l|l||}
\hline \multicolumn{2}{|c|}{} & Non complicated & Complicated & \multicolumn{2}{|c|}{ Chi-square test } \\
\cline { 3 - 6 } \multicolumn{2}{|c|}{} & No. $=95$ & No.=48 & $\mathrm{X}^{2} / \mathrm{t}^{*}$ & P-value \\
\hline Age & Mean \pm SD & $5837 \pm 1806$ & $64.69 \pm 13.55$ & $-2213^{*}$ & 0.028 \\
\hline Sex & $\begin{array}{c}\text { Female } \\
\text { Male }\end{array}$ & $\begin{array}{l}49(51.5 \%) \\
46(51.7 \%)\end{array}$ & $\begin{array}{l}28(58.33 \%) \\
20(37.0 \%)\end{array}$ & 2.902 & 0.08 \\
\hline HTN & Yes & $49(55.1 \%)$ & $30(55.6 \%)$ & 0.003 & 0.954 \\
\hline DM & Yes & $42(47.2 \%)$ & $40(74.1 \%)$ & 9.930 & 0.002 \\
\hline Smoking & Yes & $40(44.9 \%)$ & $13(33.3 \%)$ & 1.879 & 0.170 \\
\hline Heart disease & Yes & $28(31.5 \%)$ & $14(25.9 \%)$ & 0.496 & 0.481 \\
\hline
\end{tabular}

Table (9): Shows the Relation between complication and causes of ICU admission of the studied population. 


\begin{tabular}{|l|c|c|c|c|c|c|}
\hline \multirow{2}{*}{ Cause of ICU admission } & \multicolumn{2}{|c|}{ Non complicated } & \multicolumn{2}{c|}{ Complicated } & \multicolumn{2}{c|}{ Chi-square test } \\
\cline { 2 - 8 } & No. & $\%$ & No. & $\%$ & X2 & P-value \\
\hline Brain tumor & 2 & $2.2 \%$ & 2 & $3.7 \%$ & 0.262 & 0.608 \\
\hline Bronchial asthma & 3 & $3.4 \%$ & 0 & $0.0 \%$ & 1.859 & 0.173 \\
\hline Cancer stomach & 1 & $1.1 \%$ & 0 & $0.0 \%$ & 0.611 & 0.434 \\
\hline Chest infection & 25 & $28.1 \%$ & 10 & $20.8 \%$ & 0.039 & 0.843 \\
\hline Diabetic ketoacidosis & 15 & $16.9 \%$ & 5 & $9.3 \%$ & 1.611 & 0.204 \\
\hline facture pelvis & 0 & $0.0 \%$ & 4 & $7.4 \%$ & 6.782 & 0.009 \\
\hline fracture femur & 0 & $0.0 \%$ & 5 & $9.3 \%$ & 8.539 & 0.003 \\
\hline gun shot & 3 & $3.3 \%$ & 0 & $0.0 \%$ & 1.231 & 0.257 \\
\hline Intracerebral hemorrhage & 11 & $12.4 \%$ & 2 & $3.7 \%$ & 3.047 & 0.081 \\
\hline Meningitis & 2 & $2.2 \%$ & 0 & $0.0 \%$ & 1.231 & 0.267 \\
\hline Post arrest & 2 & $2.2 \%$ & 0 & $0.0 \%$ & 1.231 & 0.267 \\
\hline postoperative peritonitis & 2 & $2.2 \%$ & 0 & $0.0 \%$ & 1.231 & 0.267 \\
\hline Respiratory failure type 2 & 7 & $12.9 \%$ & 1 & $1.9 \%$ & 3.800 & 0.051 \\
\hline road traffic accident & 7 & $12.9 \%$ & 0 & $0.0 \%$ & 0.611 & 0.434 \\
\hline Septic shock & 5 & $5.6 \%$ & 3 & $5.6 \%$ & 0.000 & 1.000 \\
\hline Status epileptics & 1 & $1.1 \%$ & 0 & $0.0 \%$ & 0.611 & 0.434 \\
\hline Stroke & 8 & $9.0 \%$ & 16 & $29.6 \%$ & 10.252 & 0.001 \\
\hline
\end{tabular}

Table (10): Relation between complication and (vital signs, anthropometric measures, duration of stay in ICU and fate) of the studied patients.

\begin{tabular}{|c|c|c|c|c|c|}
\hline & \multirow{2}{*}{$\begin{array}{c}\text { Non complicated } \\
\text { No. }=95\end{array}$} & \multirow{2}{*}{$\begin{array}{c}\text { Complicated } \\
\text { No. }=48\end{array}$} & \multicolumn{2}{|c|}{ Independent t-test } \\
\hline & & & & $t / X^{2 *}$ & P-value \\
\hline Heart rate & Mean \pm SD & $99.33 \pm 20.33$ & $102.87 \pm 17.30$ & -1.068 & 0.287 \\
\hline Systolic BP & Mean \pm SD & $131.24 \pm 24.72$ & $122.41 \pm 29.39$ & 1.926 & 0.056 \\
\hline Temperature $\left({ }^{\circ} \mathbf{C}\right)$ & Mean \pm SD & $38.09 \pm 0.64$ & $38.00 \pm 0.4937$ & 0.833 & 0.406 \\
\hline Respiratory rate & Mean \pm SD & $23.34 \pm 4.03$ & $25.89 \pm 4.48$ & -3.521 & 0.001 \\
\hline Weight (kg) & Mean \pm SD & $86.40 \pm 16.15$ & $96.63 \pm 19.68$ & -3.376 & 0.001 \\
\hline Height (cm) & Mean \pm SD & $150-185$ & $165.35 \pm 4.60$ & 3.906 & 0.000 \\
\hline BMI (kg/m2) & Mean \pm SD & $30.00 \pm 4.67$ & $35.44 \pm 7.4923$ & -5.360 & 0.000 \\
\hline $\begin{array}{c}\text { Duration of stay n } \\
\text { ICU (days) }\end{array}$ & Mean \pm SD & $8.31 \pm 3.12$ & $8.04 \pm 2.54$ & 0.552 & 0.582 \\
\hline Fate & $\begin{array}{c}\text { Death } \\
\text { Discharge }\end{array}$ & $\begin{array}{l}21(23.6 \%) \\
68(76.4 \%)\end{array}$ & $\begin{array}{l}28(51.9 \%) \\
26(48.1 \%)\end{array}$ & 11.913 & 0.001 \\
\hline
\end{tabular}

Table (11): Relation between complications and laboratory data of the study groups. 


\begin{tabular}{|c|c|c|c|c|c|}
\hline \multicolumn{2}{|c|}{ laboratory data } & \multirow{2}{*}{$\begin{array}{c}\text { Non complicated } \\
\text { No. }=95\end{array}$} & \multirow{2}{*}{$\begin{array}{c}\text { Complicated } \\
\text { No. }=48\end{array}$} & \multicolumn{2}{|c|}{ Independent t-test } \\
\hline & & & & $\mathbf{t} / \mathbf{Z}^{*}$ & P-value \\
\hline TLC (cells/ul) & Mean \pm SD & $14258.43 \pm 668.53$ & $16996.49 \pm 456.55$ & -2.480 & 0.014 \\
\hline Hb (mg/dl) & Mean \pm SD & $10.46 \pm 2.45$ & $9.30 \pm 2.07$ & 2.904 & 0.004 \\
\hline Platelets (k/ul) & Mean \pm SD & $223.48 \pm 39.88$ & $214.52 \pm 40.80$ & 0.648 & 0.518 \\
\hline Urea (mg/dl) & Median & $51(36-40)$ & $47.5(39-50)$ & $-0.058^{*}$ & 0.953 \\
\hline creatinine(mg/dl) & Median & $1(0.8-1.0)$ & $1.1(0.9-1.1)$ & $-1.730 *$ & 0.084 \\
\hline$\overline{\operatorname{AST}(\mathrm{U} / \mathrm{L})}$ & Median & $29(23-30)$ & $37(29-34)$ & $-2.054 *$ & 0.040 \\
\hline ALT (U/L) & Median & $37(26-33)$ & $40.5(33-43)$ & $-1.829 *$ & 0.067 \\
\hline PH & Mean \pm SD & $7.33 \pm 0.12$ & $7.31 \pm 0.15$ & 1.002 & 0.318 \\
\hline Pco2 & Mean \pm SD & $40.89 \pm 3.01$ & $36.76 \pm 3.27$ & 1.826 & 0.070 \\
\hline HCO3 (mmol/L) & Mean \pm SD & $19.76 \pm 5.56$ & $19.00 \pm 5.75$ & 0.783 & 0.435 \\
\hline $\mathrm{Na}(\mathrm{mEq} / \mathrm{L})$ & Mean \pm SD & $133.58 \pm 6.53$ & $134.30 \pm 8.99$ & -0.547 & 0.585 \\
\hline $\bar{K}(\mathbf{m E q} / \mathbf{L})$ & Mean \pm SD & $4.01 \pm 1.02$ & $3.89 \pm 0.98$ & 0.723 & 0.471 \\
\hline Mg (mEq/L) & Mean \pm SD & $2.14 \pm 0.42$ & $1.87 \pm 0.41$ & 2.837 & 0.005 \\
\hline $\mathrm{Ca}(\mathrm{mEq} / \mathrm{L})$ & Mean \pm SD & $9.39 \pm 0.21$ & $9.37 \pm 0.49$ & 0.263 & 0.793 \\
\hline $\begin{array}{c}\text { F. cholesterol } \\
(\mathrm{mg} / \mathrm{dl})\end{array}$ & Mean \pm SD & $205.66 \pm 21.59$ & $216.11 \pm 26.41$ & -2.576 & 0.011 \\
\hline LDL (mg/dl) & Mean \pm SD & $139.20 \pm 28.29$ & $142.13 \pm 25.24$ & -0.624 & 0.533 \\
\hline Triglyceride (mg/dl) & Median & $180(155-200)$ & $180.5(160-200)$ & $-0.856^{*}$ & 0.392 \\
\hline INR & Mean \pm SD & $1.26 \pm 0.16$ & $1.28 \pm 0.14$ & -0.213 & 0.831 \\
\hline
\end{tabular}

Table (12): Relation between complications and ECG findings of the study groups.

\begin{tabular}{|c|c|c|c|c|c|}
\hline & \multirow{2}{*}{\begin{tabular}{|l} 
Non complicated \\
No. $=95$
\end{tabular}} & \multirow{2}{*}{$\begin{array}{l}\text { Complicated } \\
\text { No. }=48\end{array}$} & \multicolumn{2}{|c|}{ Chi-square test } \\
\hline & & & & $\mathrm{X}^{2} / \mathrm{t}^{*}$ & P-value \\
\hline Rate & Mean \pm SD & $98.20 \pm 20.55$ & $105.37 \pm 20.23$ & $-2.034 *$ & 0.044 \\
\hline Rhythm & $\begin{array}{l}\text { Irregular } \\
\text { Regular }\end{array}$ & $\begin{array}{l}0(0.0 \%) \\
89(100.0 \%)\end{array}$ & $\begin{array}{l}27(50.0 \%) \\
27(50.0 \%)\end{array}$ & 20.736 & 0.008 \\
\hline Ischemia & $\begin{array}{l}\text { No } \\
\text { Yes }\end{array}$ & $\begin{array}{l}65(73.0 \%) \\
24(27.0 \%)\end{array}$ & $\begin{array}{l}38(70.4 \%) \\
16(29.6 \%)\end{array}$ & 0.118 & 0.731 \\
\hline
\end{tabular}

Table (13): Shows relation between complications and echocardiographic findings in the study groups. \begin{tabular}{|l|l|l|}
\hline Non complicated & Complicated & Independent t-test \\
\hline
\end{tabular} 


\begin{tabular}{|c|c|c|c|c|c|}
\hline & & $\mathrm{No}=95$ & $\overline{N o .}=48$ & $\mathrm{t} / \mathrm{X}^{2^{*}}$ & P-value \\
\hline Aortic root $(\mathrm{cm})$ & Mean \pm SD & $2.48 \pm 0.25$ & $2.46 \pm 0.34$ & 0.396 & 0.693 \\
\hline left atrium $(\mathrm{cm})$ & Mean $\pm 3 \mathrm{D}$ & $3.70 \pm 0.44$ & $3.86 \pm 0.533 .2$ & -1.878 & 0.063 \\
\hline $\mathrm{EF}(\%)$ & Mean $\pm 3 \mathrm{D}$ & $56.09 \pm 9.15$ & $57.26 \pm 7.88$ & -0.780 & 0.437 \\
\hline RWMA & $\begin{array}{l}\text { No } \\
\text { Yes }\end{array}$ & $\begin{array}{l}59(66.3 \%) \\
30(33.7 \%)\end{array}$ & $\begin{array}{l}40(74.1 \%) \\
14(25.9 \%)\end{array}$ & $0.955^{*}$ & 0.328 \\
\hline Diastolic & Grade 1 & $53(59.5 \%)$ & $20(54 \%)$ & $10.938^{*}$ & 0.165 \\
\hline Dysfunction & $\begin{array}{l}\text { Grade } 2 \\
\text { Normal }\end{array}$ & $\begin{array}{c}15(17 \%) \\
21(23.5 \%)\end{array}$ & $\begin{array}{c}21(38.8 \%) \\
13(24 \%)\end{array}$ & & \\
\hline Mitral valve & \begin{tabular}{|c|} 
Mild MR \\
Moderate MR \\
Normal Trivial \\
MR \\
\end{tabular} & $\begin{array}{c}41(46.1 \%) \\
6(6.7 \%) \\
34(38.2 \%) \\
8(9.0 \%) \\
\end{array}$ & $\begin{array}{c}27(50.0 \%) \\
7(13.0 \%) \\
10(18.5 \%) \\
10(18.5 \%) \\
\end{array}$ & $8.197 *$ & 0.042 \\
\hline Aortic valve & \begin{tabular}{|c|} 
Calcific \\
Mild AR \\
Moderate AR \\
Normal Trivial \\
AR \\
\end{tabular} & $\begin{array}{c}14(15.7 \%) \\
0(0.0 \%) \\
3(3.4 \%) \\
71(79.8 \%) \\
1(1.1 \%)\end{array}$ & $\begin{array}{c}5(9.3 \%) \\
3(5.6 \%) \\
2(3.7 \%) \\
42(77.8 \%) \\
2(3.7 \%)\end{array}$ & 7.098 & 0.131 \\
\hline Pulmonary valve & $\begin{array}{c}\text { Mild PR } \\
\text { Normal } \\
\end{array}$ & $\begin{array}{c}2(2.2 \%) \\
87(97.8 \%)\end{array}$ & $\begin{array}{c}0(0.0 \%) \\
54(100.0 \%)\end{array}$ & $1.231^{*}$ & 0.267 \\
\hline Right Ventricle & Dilated Normal & $\begin{array}{c}12(13.5 \%) \\
77(86.5 \%)\end{array}$ & $\begin{array}{l}10(18.5 \%) \\
44(81.5 \%)\end{array}$ & $0.655^{*}$ & 0.418 \\
\hline Tricuspid valve & $\begin{array}{c}\text { Normal } \\
\text { Mild TR } \\
\text { Moderate TR } \\
\text { Severe TR } \\
\text { Trivial TR }\end{array}$ & $\begin{array}{c}45(50.6 \%) \\
14(15.7 \%) \\
2(2.2 \%) \\
1(1.1 \%) \\
27(30.3 \%) \\
\end{array}$ & $\begin{array}{c}15(27.7 \%) \\
11(20.3 \%) \\
8(14.8 \%) \\
3(5.5 \%) 17 \\
(31.4 \%)\end{array}$ & $11.632 *$ & 0.020 \\
\hline $\mathrm{Pa} \operatorname{Pr}(\mathrm{mmhg})$ & Mean \pm SD & $29.83 \pm 9.87$ & $33.81 \pm 13.03$ & -2.069 & 0.040 \\
\hline
\end{tabular}

Table (14): Relation between the three major complications (AF, PE and DVT) and Age, DM, RR and anthropometric measures

\begin{tabular}{|c|c|c|c|c|c|c||}
\hline \hline \multirow{2}{*}{ Type of complications } & AF & $\begin{array}{c}\text { Pulmonary } \\
\text { embolism }\end{array}$ & DVT & \multicolumn{2}{c|}{$\begin{array}{c}\text { One Way } \\
\text { ANOVA }\end{array}$} \\
\cline { 3 - 7 } & No.=27 & No.=13 & No.=8 & F/X ${ }^{2 *}$ & P-value \\
\hline Age (yrs) & Mean \pm SD & $61.77 \pm 7.92$ & $68.74 \pm 14.61$ & $64.25 \pm 6.88$ & 1.558 & 0.222 \\
\hline DM & $\begin{array}{c}\text { No } \\
\text { Yes }\end{array}$ & $\begin{array}{c}3(23.1 \%) \\
10(76.9 \%)\end{array}$ & $\begin{array}{c}9(33.3 \%) \\
18(66.7 \%)\end{array}$ & $\begin{array}{c}1(12.5 \%) \\
7(87.5 \%)\end{array}$ & $1.501^{*}$ & 0.472 \\
\hline $\begin{array}{c}\text { RR (train) } \\
\text { Mean } \pm \text { SD }\end{array}$ & $27.31 \pm 3.20$ & $25.22 \pm 5.0318-$ & $24.38 \pm 3.62$ & 1.386 & 0.261 \\
\hline $\begin{array}{c}\text { Weight } \\
(\mathrm{kg})\end{array}$ & Mean \pm SD & $109.85 \pm 20.75$ & $87.78 \pm 15.26$ & $113.75 \pm 14.48$ & 11.761 & 0.000 \\
\hline $\begin{array}{c}\text { Height } \\
(\mathrm{cm})\end{array}$ & Mean \pm SD & $165.31 \pm 2.98$ & $165.63 \pm 5.46$ & $166.00 \pm 4.24$ & 0.054 & 0.948 \\
\hline $\begin{array}{c}\text { BMI } \\
(\mathrm{kg} / \mathrm{m} 2)\end{array}$ & Mean \pm SD & $40.82 \pm 7.49$ & $32.05 \pm 5.8623-$ & $41.15 \pm 6.84$ & 11.110 & 0.000 \\
\hline
\end{tabular}

Table (14) continues: 


\begin{tabular}{|c|c|c|c|c|c|c|}
\hline \multirow{2}{*}{\multicolumn{2}{|c|}{ Type of complications }} & \multirow{3}{*}{\begin{tabular}{|c|} 
AF \\
No. $=27$ \\
$18(66.7 \%)$ \\
$9(33.3 \%)$ \\
\end{tabular}} & \multirow{3}{*}{\begin{tabular}{|r|} 
DVT \\
No. $=8$ \\
$3(37.5 \%)$ \\
$5(62.5 \%)$
\end{tabular}} & \multirow{3}{*}{\begin{tabular}{|r|}
$\begin{array}{l}\text { Pulmonary } \\
\text { embolism }\end{array}$ \\
No. $=13$ \\
$6(46.2 \%)$ \\
$7(53.8 \%)$ \\
\end{tabular}} & \multicolumn{2}{|c|}{\begin{tabular}{|l|} 
Chi-squares \\
test \\
\end{tabular}} \\
\hline & & & & & $\mathrm{X}^{2} / \mathrm{F}^{*} / \mathrm{K} \bullet$ & \\
\hline Fate & $\begin{array}{c}\text { Death } \\
\text { Discharge }\end{array}$ & & & & 2.872 & 0.238 \\
\hline $\begin{array}{c}\text { FLC } \\
\text { (cells/ill) }\end{array}$ & Mean \pm SD & $\begin{array}{l}16089.27 \\
\pm 8330.56\end{array}$ & $\begin{array}{c}19912.50 \pm 3570 \\
69\end{array}$ & $\begin{array}{l}17492.31 \\
\pm 8712.30\end{array}$ & $0.745^{*}$ & 0.480 \\
\hline $\mathrm{Hb}(\mathrm{mg} / \mathrm{dl})$ & Mean \pm SD & $10.08 \pm 2.04$ & $7.68 \pm 0.92$ & $8.38 \pm 1.70$ & $7.266^{*}$ & 0.002 \\
\hline$\overline{\mathrm{AST}(\mathrm{U} / \mathrm{L})}$ & Median & $40(31-40)$ & $31.5(30-40.5)$ & $30(28-30)$ & $2.469 *$ & 0.291 \\
\hline$\overline{\mathrm{ALT}(\mathrm{U} / \mathrm{L})}$ & Median & $44(30-50)$ & $40(37.5-47.5)$ & $36(30-50)$ & $0.293^{*}$ & 0.864 \\
\hline $\begin{array}{c}\mathrm{Mg} \\
(\mathrm{mEq} / \mathrm{L})\end{array}$ & Mean \pm SD & $1.94 \pm 0.31$ & $1.79 \pm 0.34$ & $1.97 \pm 0.31$ & $0.237^{*}$ & 0.790 \\
\hline $\begin{array}{c}\text { Total } \\
\text { cholesterol } \\
(\mathrm{mg} / \mathrm{dl})\end{array}$ & Mean \pm SD & $212.30 \pm 24.62$ & $222.50 \pm 18.03$ & $227.31 \pm 20.16$ & $2.128 *$ & 0.131 \\
\hline
\end{tabular}

Table (15): Relation between three major complications (AF,PE \& DVT) and Fate, TLC, Hb, AST, ALT, Mg, T. cholesterol

\begin{tabular}{|c|c|c|c|c|c|c|c|}
\hline \multirow{2}{*}{} & \multicolumn{5}{|c|}{ Univariate regression analysis } & \multicolumn{2}{|l|}{ Multivariate regression analysis } \\
\cline { 2 - 9 } & B & S.E. & Wald & P-value & $\begin{array}{c}\text { Odds ratio (OR) [95\% } \\
\text { Cl] }\end{array}$ & P-value & $\begin{array}{c}\text { Odds ratio (OR) [95\% } \\
\text { Cl] }\end{array}$ \\
\hline Age & 0.024 & 0.011 & 4.641 & 0.031 & $1.025(1.002-1.048)$ & 0.238 & $1.037(0.976-1.101)$ \\
\hline DM & 1.162 & 0.376 & 9.546 & 0.002 & $3.197(1.530-6.683)$ & 0.318 & $0.473(0.109-2.056)$ \\
\hline RR & 0.140 & 0.043 & 10.735 & 0.001 & $1.150(1.058-1.251)$ & 0.001 & $1.528(1.196-1.951)$ \\
\hline WT & 0.033 & 0.010 & 9.838 & 0.002 & $1.033(1.012-1.055)$ & 0.704 & $0.979(0.880-1.090)$ \\
\hline Height & -0.122 & 0.034 & 12.646 & 0.001 & $0.885(0.828-0.947)$ & 0.058 & $0.862(0.740-1.005)$ \\
\hline BM1 & 0.155 & 0.035 & 19.383 & 0.001 & $1.167(1.090-1.250)$ & 0.018 & $1.423(1.061-1.907)$ \\
\hline TLC & 0.001 & 0.001 & 5.481 & 0.019 & $1.000(1.000-1.000)$ & 0.075 & $1.000(1.000-1.000)$ \\
\hline HB & -0.223 & 0.081 & 7.682 & 0.006 & $0.800(0.683-0.937)$ & 0.689 & $0.941(0.700-1.266)$ \\
\hline AST & 0.002 & 0.002 & 0.742 & 0.389 & $1.002(0.998-1.005)$ & & \\
\hline ALT & 0.003 & 0.002 & 1.626 & 0.202 & $1.003(0.998-1.008)$ & & \\
\hline Mg & -0.904 & 0.332 & 7.409 & 0.006 & $0.405(0.211-0.776)$ & 0.000 & $0.043(0.008-0.223)$ \\
\hline T.cholestrol & 0.020 & 0.008 & 6.140 & 0.013 & $1.020(1.004-0.036)$ & 0.544 & $0.991(0.962-1.021)$ \\
\hline
\end{tabular}

\section{Discussion:}


There was statistically significant difference regarding age. The range of age of the study group was from 17 to 93 years, the mean age in complicated group was around 64.69 years old, compared with mean of 58.37 years in non-complicated group. This is consistent with Guenancia et al. ${ }^{(5)}$.

There was no statistically significant difference regarding sex. However, the number of females with complications was higher than number of males. This is consistent with Fuster study ${ }^{(3)}$. There was no statistically significant difference regarding smoking. There was no statistically significant difference regarding hypertension. However the number of hypertensive patients with complications was higher than normotensive patients. There was statistically significant difference regarding Diabetes Mellitus. However the number of diabetic patients with complications was 40 patients $(74.1 \%$ of cases) and the number of non-diabetic patients with complications were 14 patients (25.9\% of cases). This is consistent with Rosenberg study (6). There was no statistically significant difference regarding heart failure. As regard causes of admission to ICU there was statistically significant difference regarding Cerebro-Vascular Stroke, however the number of patients of cerebroVascular Stroke with complications was 16 patients (29.6\% of all complicated patients). This is consistent with Slipman study ${ }^{(7)}$. There was statistically significant difference regarding fracture pelvis, patients admitted due to fracture pelvis were 4 patients, all of them had cardiac complications. This is consistent with Pedro study ${ }^{(8)}$.

There was statistically significant difference regarding fracture femur. This is consistent with Kim study ${ }^{(4)}$. There was no statistically significant difference regarding chest infection ( $<\mathrm{P}$-value 0.05$)$. There was statistically significant difference regarding weight. This is consistent with Stein study ${ }^{(9)}$.

There was statistically significant difference regarding BMI . In the complicated group the range of BMI was from 23 to 50 $\mathrm{kg} / \mathrm{m} 2$ with mean around $35.4 \mathrm{~kg} / \mathrm{m} 2$, compared with range of 18 to $46 \mathrm{~kg} / \mathrm{m} 2$ in the noncomplicated group with mean around $30 \mathrm{~kg} / \mathrm{m} 2$. This is was consistent with Stein et al ${ }^{(9)}$.

There was a statistically significant relation found between complications and respiratory rate with. This is was consistent with Torbicki Astein et al ${ }^{(10)}$. There was statistically significant difference regarding fate of the patients in the study population. In the complicated group the number of deaths was 28 patients and in non-complicated group the number of deaths was only 21 patients. There was statistically significant difference regarding TLC. The mean range of TLC in complicated group was 17.000 cells/ul. There was statistically significant difference regarding hemoglobin level. However patients with complications their $\mathrm{Hb}$ level was less than $10 \mathrm{mg} / \mathrm{dl}$. This is was consistent with Jiménez D et al (11). There was statistically significant relation found between complicated patients and AST level, total cholesterol and heart rate. There was statistically significant difference regarding mitral valve. There was a statistically significant relation between complicated patients and pulmonary artery pressure. There was statistically significant relation found between body weight and the incidence of DVT.The weight in $\mathrm{kg}$ in patients complicated with DVT was $113 \mathrm{~kg}$, while the mean body weight in non-complicated group was $86 \mathrm{~kg}$. Venous thromboembolism risk factors are more specific to critically ill patient; age, immobilization, obesity, past history of personal or familial VTE, and past history of neoplasm ${ }^{(2)}$.

There was a significantly important relation between the DVT and increased BMI, however the body mass index in DVT complicated group was $41.1 \mathrm{~kg} / \mathrm{m} 2$ (class 3 obesity). There was a significantly important relation between the hemoglobin level and the incidence of DVT. However the hemoglobin level in DVT group was $7.6 \mathrm{mg} / \mathrm{dl}{ }^{(11)}$.

\section{Conclusion}

The study concluded that, the ICU patients have a higher risk than other patients for adverse cardiac complications like AF, DVT and pulmonary embolism.Cardiac complications lead to increased rate of mortality and morbidity.

\section{References:}

1. Boonyawat K, Crowther M (2015) : Venous thromboembolism prophylaxis in critically ill patients Semin Thromb Hemost., 41 (1) : 068-074.

2. Cook D, Crowther M, Meade M et al. (2005): Deep venous thrombosis in medical-surgical critically ill patients: prevalence, incidence, and risk factors. 
Critical care medicine, 33 (7): 15065 1571.

3. Fuster V, Lars E, Cannom $\mathrm{H}$ et al. (2011) : Management of patients with atrial fibrillation. Journal of the American College of Cardiology, 57 (11) : 101-198.

4. Kim H, Shin A, Chang H, Dong-Woo $S$ and Won Young Kim (2016): Timing of pulmonary embolisms in femur fracture patients: Incidence and outcomes. Journal of Trauma and Acute Care Surgery, 80 (6): 952-956.

5. Guenancia C, Christine B, Gabriel L, Sandrine V, Rémi B,Sébastien P, Arnaud P, Pierre-Emmanuel C, Jean-P (2015) :Incidence and predictors of new-onset atrial fibrillation in septic shock patients in a medical ICU. https://www.ncbi.nlm.nih.gov/pmc/arti cles/PMC4428753/

6. Rosenberg M, Warren M (2012): Diastolic dysfunction and risk of atrial fibrillation. Circulation, 126 (19): 2353-2362.

7. Slipman W, Jason S, Lipetz H, Jackson J (2000): Deep venous thrombosis and pulmonary embolism as a complication of bed rest for low back pain. Archives of physical medicine and rehabilitation, 81(1) : 127-129.

8. Pedro, Jose $\mathbf{R}$ Caeiro, Rocío C, Angela M, Samuel S and Manuel M (2014): Complications of hip fractures: A review. World journal of orthopedics, 5 (4): 402-411.

9. Stein, D, Fadi M and Jose G (2011): Obesity and pulmonary embolism: the mounting evidence of risk and the mortality paradox. Thrombosis research, 128 (6): 518-523.

10. Torbicki A, Konstantinides S, Giancarlo A, Nicolas D, David F, Nazzareno G, Simon R(2014) : ESC guidelines on the diagnosis and management of acute pulmonary embolism. Polish Heart Journal, 72 (11) : 997-1053.

11. Jiménez D, Carlos E, David $M$, Gema D, Jesús C, Ángel A, Roger D (2009) : Association of anaemia and mortality in patients with acute pulmonary embolism. Thrombosis and haemostasis, 102 (1) : 153-158. 